\title{
The Origin of the Elements
}

\author{
S. R. Lesher and A. Arend \\ Department of Physics, University of Wisconsin - La Crosse
}

\begin{abstract}
There are about 35 nuclei found in nature, which are not susceptible to neutron capture and are explained by the p-process. The modeling for this process requires thousands of nuclear reactions involving both stable and unstable nuclei including $(\alpha, \alpha),(\alpha, p)$ and $(\alpha, \gamma)$ reactions. In a recent experiment, the cross section of the reaction ${ }^{120} \mathrm{Te}(\alpha, \mathrm{p}){ }^{123} \mathrm{I}$ was measured in the energy range of astrophysical interest for the p-process. The $\alpha$ beam from the Notre Dame FN Tandem Van de Graaff accelerator bombarded highly enriched self-supporting ${ }^{120} \mathrm{Te}$ targets and the $\gamma$-rays from the activated ${ }^{123} \mathrm{I}$ was counted with a pair of Ge clover detectors in close geometry.
\end{abstract}

\section{Introduction}

We have understood the basic processes of stellar burning, and the ability to produce the lightest of elements, but moving beyond the simple model has proven difficult to complete. Since the pioneering work of Burbidge, Burbidge, Fowler, and Hoyle in 1957 ( $\left.\mathrm{B}^{2} \mathrm{FH}\right)$ [1], which set up the framework for nucleosynthesis, there has been steady progress in the quest to observe the elemental abundances in terms of the nuclear processes which take place in stars. In recent years, the progress has rapidly increased with the advancement in observational capabilities over a varied range of the electromagnetic spectrum, development of radioactive beam facilities, advance detection techniques, and computing power which allow the study of nuclear properties previously inaccessible. The next generation of radioactive beam facilities is planned to access even more regions of interest but is years from completion. At this time, there is still work in accessible regions that can be studied.

Decades of dedicated research have produced a detailed version of atomic structure. Each atom consists of a small nucleus, about $10^{-12}$ to $10^{-13} \mathrm{~cm}$ in diameter, surrounded by a cloud of negatively charged electrons. This nucleus is only about 1/20,000 the diameter of the atom! Even though the nucleus is small, it can contain hundreds of positively charged protons and uncharged neutrons, referred to collectively as nucleons. To express a specific nucleus, we explicitly denote its composition. The atomic number, $\mathrm{Z}$, equals the number of protons, $\mathrm{N}$ denotes the number of neutrons, and the mass number $A$ is equal to the number of nucleons $(\mathrm{N}+\mathrm{Z})$. Isotopes, nuclei with the same $\mathrm{Z}$ but different $\mathrm{N}$ and $\mathrm{A}$ values, are symbolically expressed as ${ }_{Z}^{A} X$ where $\mathrm{X}$ represents the chemical symbol for the element. For example, carbon is a common element. The most naturally abundant carbon isotope makes up $98.9 \%$ of all carbon atoms and is denoted by ${ }^{12} \mathrm{C}$; however, the isotope ${ }^{13} \mathrm{C}$ has an extra neutron and comprises only $1.1 \%$ of all carbon atoms. There are still 13 additional isotopes of carbon, which decay in some period of time (i.e., are not stable) with a characteristic half-life $\left(\mathrm{t}_{1 / 2}\right)$, which ranges from nanoseconds to years. For historical reasons, some isotopes are denoted by special names; ${ }^{1} \mathrm{H},{ }^{2} \mathrm{H}$ and ${ }^{3} \mathrm{H}$ are called the proton $(\mathrm{p})$, deuteron (d), and triton ( $\mathrm{t}$ ), respectively, and the nucleus of the ${ }^{4} \mathrm{He}$ isotope is called an $\alpha$-particle $(\alpha)$.

Particles release energy in uniting to form an atomic nucleus. The energy given up in this formation is called the binding energy, which is the difference in mass energy between a nucleus ${ }_{Z}^{A} X$ and its constituent $\mathrm{X}$ protons and $\mathrm{N}$ neutrons: 


$$
B=\left[Z m_{p}+N m_{n}-m\left({ }^{A} X\right)\right]^{2}
$$

where $m_{p}$ and $m_{n}$ are the mass of the proton and neutron, respectively, and $c^{2}$ is the speed of light squared given as a conversion factor $931.50 \mathrm{MeV} / \mathrm{u}$. This is the beginning of the fuel cycle which takes place in the interior of stars and the first step in the origin of the elements.

The work of Burbidge et al. proposed a variety of nuclear processes that take place in the interiors of stars to produce the observed elemental and nuclidic abundances. The abundance of an isotope measures how relatively common it is, or how much of that isotope is in the universe by comparison to all other isotopes. Since 1957, the cosmic abundance curves have been modified with new information, but the nuclear processes proposed are still the basis of what we know about nucleosynthesis. To explain all the features of the original abundance curves, Burbidge et al. viewed the elements as evolving through at least eight processes, where only hydrogen is created in the formation of the universe. It has since been grouped into the following processes [1][2][3]:

- hydrogen burning

- helium burning

- heavier element burning

- the s-, r- and p-processes

- the 1-process

The first three processes are in the interior of stars and explain the synthesis of isotopes from helium to iron. Hydrogen burning converts hydrogen to helium; helium burning converts helium to carbon, oxygen and so on. The chain starts as follows:

$$
\begin{aligned}
& { }^{1} \mathrm{H}+{ }^{1} \mathrm{H} \rightarrow{ }^{2} \mathrm{H}+e^{+}+v \\
& { }^{2} \mathrm{H}+{ }^{1} \mathrm{H} \rightarrow{ }^{3} \mathrm{He}+\gamma \\
& { }^{3} \mathrm{He}+{ }^{3} \mathrm{He} \rightarrow{ }^{4} \mathrm{He}+2^{1} \mathrm{H}
\end{aligned}
$$

where $\mathrm{e}^{+}$is a positron and $\mathrm{v}$ is a neutrino.

Heavier element burning includes carbon, oxygen and neon burning which produces elements $16<A<28$ and silicon burning which is responsible from the elements $25 \leq A \leq 60$. These cycles cannot produce significant amounts of heavier elements, partly because of high Coulomb barriers for the reactions; therefore, elements higher than Fe are mainly produced by neutroncapture, the s- and r-process.

Neutron capture is a nuclear reaction in which a neutron collides with a nucleus and merge to form a heavier nucleus. When the neutron flux is small, the nucleus captures a neutron and then decays before capturing another neutron. Nuclearsynthesis takes place in the interior of stars where there is a high neutron flux density. In these cases, the nucleus is bombarded with neutrons and may or may not have time to decay before it captures another neutron. The s- and rprocess are compared to beta-decay ( $\beta$-decay) where the mass number rises by a large amount while the atomic number stays the same. 
The process in which neutron capture is slow compared to beta decay is the slow or s-process. The lighter processes which produce nuclide up to iron liberate neutrons which are then used with pre-existing "seed" nuclide to produce the s-process nuclide. This secondary process can explain elements heavier than bismuth [4]. The r-process (rapid neutron capture) is a violent process, occurring in supernovae or disruptions in neutron stars. This process is responsible for the synthesis of uranium from seed nuclei and other higher elements. There are still about 35 nuclei found in nature, which are not susceptible to neutron capture and therefore cannot be explained by the r- or s-process. The p-process makes their existence possible [4].

The p-process nuclei are rare, more proton rich, and less abundant than nuclei produced by other means. It is this rare reaction, which has been harder to grasp by the community and about which more information is needed. One possible location of this process is far from the core collapse center in the layers of a type II supernova explosion. Under these high temperature, high-density conditions, seed nuclei from the r-and s-nuclei are photodisintegrated $(\gamma, n)$, and the nuclei are shifted to the proton rich side of the valley of stability. Every time a neutron is lost, the neutron binding energy is increased, and the process becomes less likely. If the temperatures are high enough, the process can continue with $(\gamma, p)$ - and $(\gamma, \alpha)$ - reactions to lower masses. The production rates of the p-process nuclei are still relatively unknown.

The astrophysical rates for the p-process reactions are evaluated from the Hauser-Feshbach (HF) statistical model for the neutron and $\alpha$-particle captures, and then the reverse photodisintegration rates are obtained. This is not a simple process. Many input parameters must be known, including proper optical potentials, ground state properties (masses, deformation, matter densities) of both the target and residual nuclei, excited states, and when these are not know experimentally, nuclear mass models are used [5]. The HF model uses the $\alpha$-particle capture and requires the structure of the nuclei to be known. Recent simulations demonstrate the importance of these $(\gamma, \alpha)$ reactions on the overall reaction flow and therefore abundance of the $p$ nuclei [6]. These simulations indicate ${ }^{120} \mathrm{Te}$ is under produced in comparison to observations [6]. Previous experiments ${ }^{120} \mathrm{Te}(\mathrm{p}, \gamma){ }^{121} \mathrm{I}$ and ${ }^{120} \mathrm{Te}(\mathrm{p}, \mathrm{n}){ }^{120} \mathrm{I}$ have already been completed [7] to begin to address these issues. In this set of experiments, we set out to explore the ${ }^{120} \mathrm{Te}(\alpha, \mathrm{p}){ }^{123}$ I reaction.

\section{Experiment and Results}

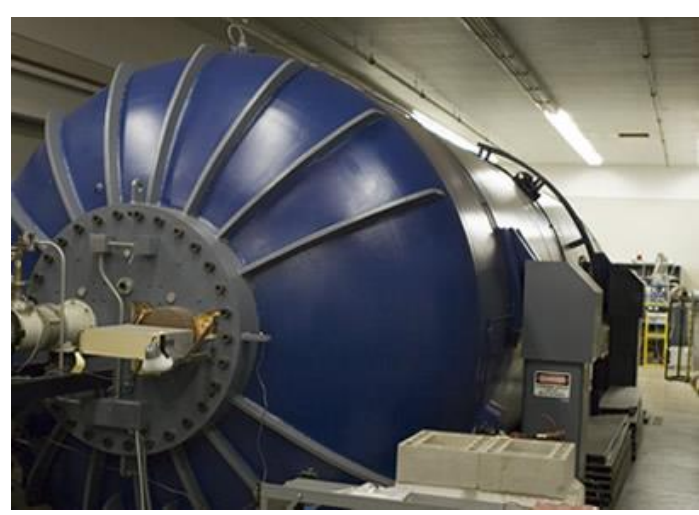

Figure 1: The University of Notre Dame FN Tandem accelerator.
The experiments took place at the University of Notre Dame's Institute for Structure \& Nuclear Astrophysics (ISNAP). The ND FN Tandem Van de Graaff accelerator (Fig. 1) is capable of terminal voltages over 10.6 MV with beam energies from a few $\mathrm{MeV}$ up to more than $100 \mathrm{MeV}$.

In the first set of experiments, a series of targets were analyzed to account for their composition using Rutherford Backscattering. Rutherford Backscattering is a high kinetic energy elastic collision between an incident ion beam and a target particle. ${ }^{12} \mathrm{C}^{3+}$ ions were accelerated to $15 \mathrm{MeV}$ using the tandem FN accelerator and impinged on each of the targets in 
question. Since the targets are made of thin foil, there is a chance of interaction and scattering by Coulomb collision. The chance of scattering depends upon the atomic number and isotope (among other things) and therefore, we can use this cross-section of the reaction to characterize the isotopic composition of each target of interest. The outgoing $\alpha$ particles were measured with a silicon detector at $150^{\circ}$ with respect to the beam axis.

The Rutherford Backscattering (RBS) experiments allowed the identification of the targets and the thickness which is necessary for the use in the following reaction experiment. Fig. 2 shows the data from a sample of Tellurium which was determined to be $555 \mu \mathrm{g} / \mathrm{cm}^{2}$ thick ${ }^{120} \mathrm{TeO}_{2}$. In this figure, two alpha peaks are clearly shown, which corresponds to the known energies of ${ }^{120} \mathrm{Te}$.

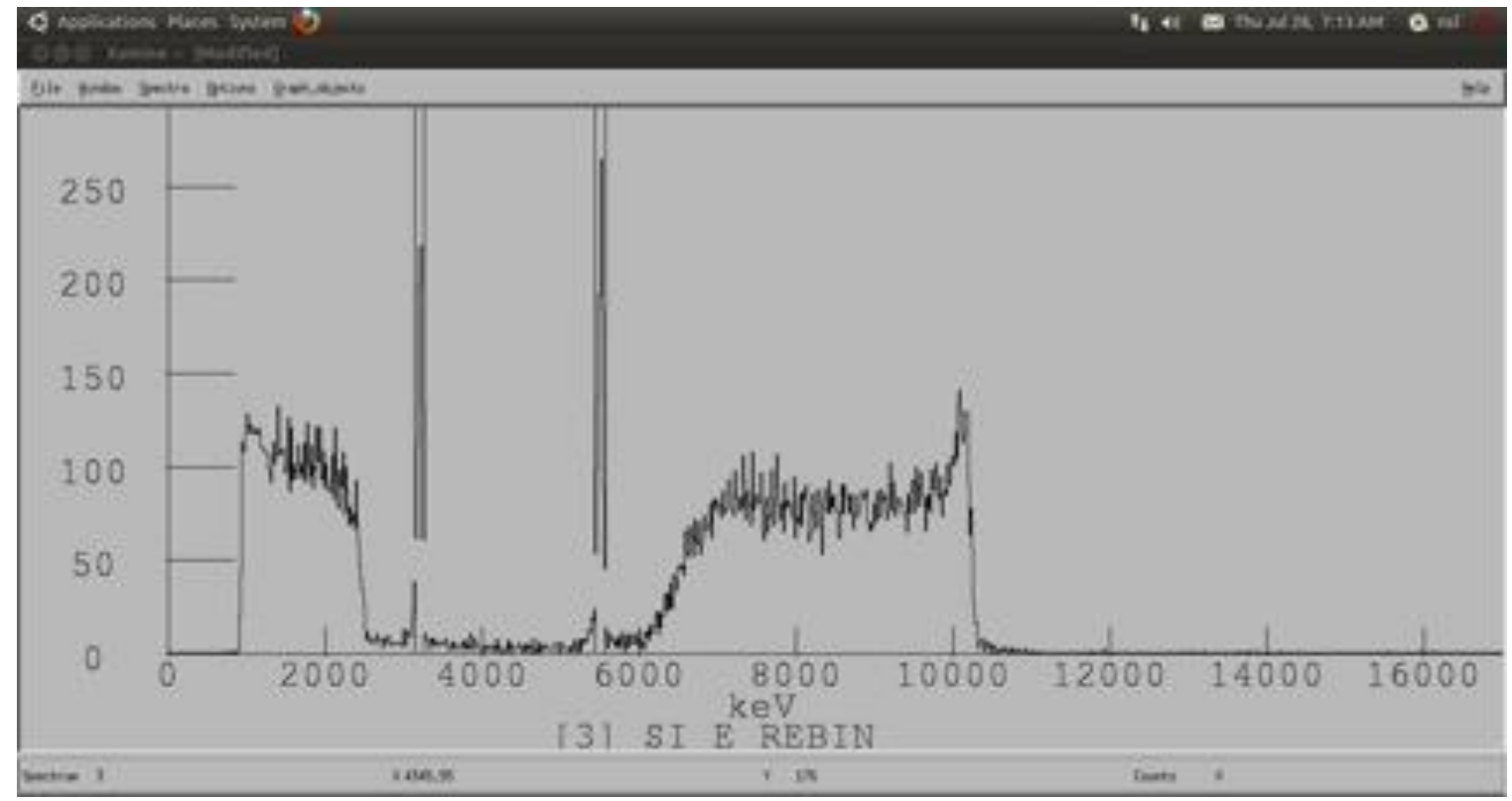

Figure 2: ${ }^{120} \mathrm{TeO}_{2}$ identified by the two alpha peaks shows. Alpha energy is shown on the $\mathrm{x}$-axis in keV and number of counts on the y-axis.

After identifying the targets of interest, the FN provided a high quality $\alpha$-beam for the ${ }^{120} \mathrm{Te}(\alpha, p){ }^{123} \mathrm{I}$ experiment. After irradiation of the target using a range of alpha-beam energies, the activated target was taken to a separate low-background counting area and the induced $\gamma$-ray activity was measured by two Clover detectors placed face-to-face in close geometry (as shown in Fig. 3). The Clover detectors consist of four high purity germanium crystals that gather information about the $\gamma$-rays which have been emitted from the decaying nucleus. This procedure has been used before at ISNAP with great success and details can be found in Ref. [7]. 


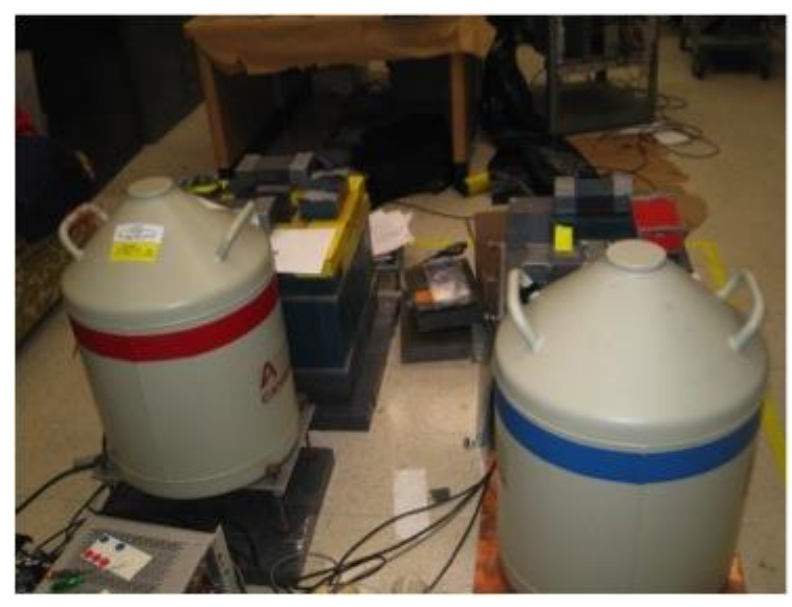

Figure 3: The detector on the left is an approximately $110 \%$ efficient HPGe detector, the one on the right is $\sim 55 \%$ efficient. This equipment was used to detect the de-exciting $\gamma$-rays after the irritation of the target by $\alpha$ particles by the ND FN Tandem Van de Graaff accelerator.

The ${ }^{120}$ Te foils were removed after irridation and transferred to one of the HPGe detectors to be counted. The cross section (Fig. 4) was determined from the $\gamma$-rays yields and compared to a code using Hauser=Feshbach statistical statistical theory called NON SMOKER.

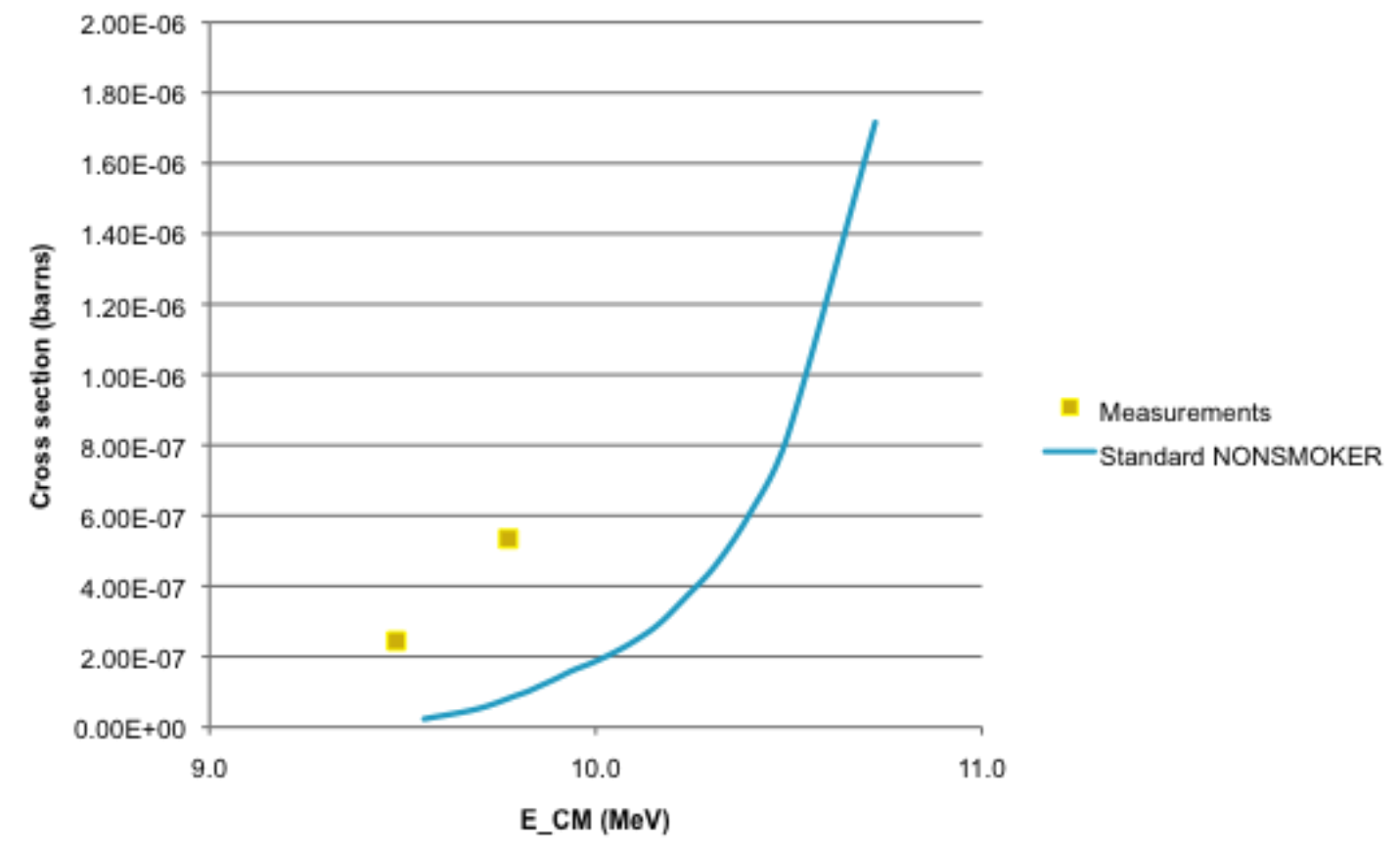

Figure 4: Comparison of the measured cross section of ${ }^{120} \mathrm{Te}(\alpha, \mathrm{p})^{123} \mathrm{I}$ to the calculated cross section using the standard 'NONSMOKER' code which uses Hauser-Feshbach (HF) statistical model theory. [Preliminary]

\section{Conclusion}

The ${ }^{120} \mathrm{Te}(\alpha, \mathrm{p})^{123} \mathrm{I}$ experiment was performed over two weeks in the summer of 2011 , with an ongoing analysis. There is still much work needed to be done, the remaining data need to be analyzed, the statistical models need to be run and an interpretation of the data discussed. This work has a far-reaching impact on the community as it can help make the HF model for the 
origin of the elements more robust, and therefore, able to predict isotopes we cannot reach in the laboratory.

\section{Acknowledgements}

The authors would like to thank their collaborators, A. Aprahamian, A. Kontos, W. P. Tan, R. T. Güray and N. Özkan and generous funding by the National Science Foundation Grant No. PHY08-22-648. SRL would also like to thank the Wisconsin Space Grant Consortium for their generous funding.

\section{Bibliography}

[1] Burbidge, E. Margaret, Burbidge, G. R., Fowler, William A., and Howyle, F. Rev. Mod. Phys., 29 (1957), 547.

[2] Rolfs, Claus E. and Rodney, Williams S. Cauldrons in the Cosmos. The University of Chicago Press, Chicago, 1988.

[3] Ehmann, William D. and Vance, Diane E. Radiochemistry \& Nuclear Methods of Analysis. John Wiley \& Sons, New York, 1991.

[4] Meyer, Bradley S. Annu. Rev. Astron. Astrophys., 32 (1994), 153.

[5] Arnould, M. and Goriely, S. Phys. Reports, 384 (2003), 1.

[6] Rapp, W., Gorres, J., Wiescher, M., Schatz, H., and Kappeler, F. Astrophys. J., 653 (2006), 474.

[7] Guray, R. T., Ozkan, N., Yalcin, C. et al. Phys. Rev. C, 80 (2009), 035804. 